\title{
The Role of Unification in Micro-Explanations of Physical Laws *
}

\author{
Erik WEBER and Merel LEFEVERE
}

Received: 06.01.2013

Final version: 02.09.2013

BIBLID [0495-4548 (2014) 29: 79; pp. 41-56]

DOI: $10.1387 /$ theoria. 7054

ABSTRACT: In the literature on scientific explanation, there is a classical distinction between explanations of particular facts and explanations of laws. This paper is about explanations of laws, more specifically about microexplanations of laws in physics. We investigate whether providing unificatory information has a surplus value in micro-explanations of physical laws. Unificatory information is information that provides ontological unification in the sense defined by Uskali Mäki. We argue that providing unificatory information may lead to explanations with more explanatory power (we use Jim Woodward's concept of explanatory power for that) and that it may lead to more strongly supported explanations.

Keywords: causal mechanisms; explanation of laws; explanatory virtues; unification.

RESUMEN: En la literatura sobre explicación científica hay una distinción clásica entre explicaciones de hechos particulares y explicaciones de leyes. El presente artículo trata de las explicaciones de las leyes, más en concreto, de las microexplicaciones de las leyes en la física. Analizamos si proporcionar información unificadora posee un valor adicional en las microexplicaciones de las leyes físicas. La información unificadora es información que proporciona unificación ontológica en el sentido definido por Uskali Mäki. Argumentamos que proporcionar información unificadora puede llevar a explicaciones con mayor poder explicativo (aquí empleamos el concepto de poder explicativo de Jim Woodward), y también a explicaciones más firmemente apoyadas.

Palabras clave: mecanismos causales; explicación de las leyes; virtudes explicativas; unificación.

\section{Introduction}

In the literature on scientific explanation, there is a classical distinction between explanations of particular facts and explanations of laws. This paper is about explanations of laws, more specifically micro-explanations of physical laws. In this introduction we first clarify two key terms: physical law and micro-explanation. Then we clarify why and how we will investigate the role of unification in micro-explanations of physical laws (i.e., we clarify our aims and our method).

\section{1}

In order to get an idea of the subject-matter involved here, let us look at some examples and important groups of physical laws (the groups are certainly not exhaustive). First, there are quantitative laws, typically expressed by means of a mathematical formula. An example is the Ideal Gas Law (IGL):

\footnotetext{
* The authors thank Koen Strijckmans, Maarten Van Dyck, the participants of the Explanation, Causality and Unification workshop and two anonymous referees for their comments on earlier versions of this paper.
} 
For equal quantities of gas in a container, the product of the pressure $P$ and the volume $V$ is proportional to the temperature $T$, with a proportionality constant $R$ (the ideal gas constant).

Second we have qualitative regularities describing sufficient condition relations between properties:

In mercury thermometers, the mercury level first drops and then rises when they are rapidly immersed in hot water.

Third, there are regularities in which capacities are ascribed to classes of objects, for instance:

Copper has the capacity to conduct electricity.

As an overall view on what scientific laws are, we adopt Sandy Mitchell's pragmatic approach $(1997,2000,2003)$. The core idea of this approach is that a continuum of contingency/stability replaces the traditional dichotomy between laws and accidental generalisations. Some generalisations are more spatio-temporally stable than others, because the conditions on which they depend are more stable in space, in time or both. For instance, the truth of Galileo's law depends on the mass of the Earth, which is quite stable in time. Biological laws (the laws that Mitchell was primarily interested in) have less spatio-temporal stability than Galileo's law (and other physical laws) but are much more stable than the classical philosopher's examples of accidental generalisations (e.g. "All coins in Goodman's pocket are made of copper"). Other important aspects of Mitchell's view are (a) that laws may have exceptions and (b) that they are not necessarily causal.

\section{2}

We use the term micro-explanation to refer to explanations of (physical) laws that invoke entities and activities at a lower level. In other words: in micro-explanations there is decomposition of the system of which the behaviour is explained. Not every explanation of a law is a micro-explanation. Take for instance:
General law:
All waves reflect.
Auxiliary hypothesis:
Sounds are waves.
Explanandum:
Sounds reflect.

In this explanation the explanandum law is subsumed under a more general law by means of an auxiliary hypothesis. This explanation does not refer to the (activities of the) entities of which sounds are composed, so it is not a micro-explanation. ${ }^{1}$

We distinguish between two types of micro-explanations: mechanistic explanations and aggregation explanations. What is a mechanistic explanation of a law? Starting with Machamer, Darden and Craver's seminal paper 'Thinking about Mechanisms' (2000) a mechanistic tradition has developed in the philosophy of explanation. For laws, mechanistic explanations are defined as follows:

\footnotetext{
${ }^{1}$ For a real example, see chapter 33 of volume II of Feynman et al. 2010; in this chapter several laws of geometrical optics (reflection, refraction) are derived from Maxwell's equations.
} 
A mechanistic explanation of a law is a description of the underlying mechanism.

We define mechanisms as follows:

A mechanism is a collection of entities and activities that are organized such that they realize the macro-level behaviour of a system.

This definition includes the three key terms which mechanists use: entities, activities and organisation. A description of a mechanism is usually called a model of the mechanism. The core idea of the mechanists is that, in order to have explanatory value, the model has to describe the mechanism in terms of its entities, its activities and the way these entities and activities are organized. We look at some characteristic quotes. Bechtel \& Abrahamsen write:

A mechanism is a structure performing a function in virtue of its component parts, component operations, and their organization. The orchestrated functioning of the mechanism is responsible for one or more phenomena. $(2005,423)$

Carl Craver writes:

$[\mathrm{M}]$ echanisms are entities and activities organized such that they exhibit the explanandum phenomenon. (2007, 6; italics removed)

These quotes show that mechanists have no unique way of defining what a mechanism is. However, there is a common core idea and our definition of a mechanism captures this core idea.

Before we turn to aggregation explanation, two remarks are useful to situate our use of the core idea of the mechanists.

(1) The first mechanists mainly focused on the biological and cognitive sciences, i.e. their aim was to develop a theory about how to explain biological and cognitive capacities. By applying mechanistic ideas to physics, we imply that these ideas are useful outside the originally intended domain. The examples we give in Section 2 of this paper support this extension. ${ }^{2}$

(2) There is an ambiguity in the mechanisms literature. They want to deal with behaviour of individual systems, but also with typical behaviour of classes of individuals (e.g. a species or a class of computing devices). For instance, they are not only interested in the structure of the answer to the question "Why does this desk calculator have the capacity to perform exact numerical calculations?" but also in the form of the answer to "Why do desk calculators have the capacity to perform exact numerical calculations?". This distinction is not always made clearly in the mechanisms literature. We will come back to this issue later. What is important now is that, given our focus on laws, we will always be talking about classes of individuals.

We use the term aggregation explanation to refer to explanations which explain the behaviour of the macro-systems (as described in the law) by means of the component parts, the activities of these parts, organisation and random distribution of behaviour of components. This means that, compared to mechanistic explanations, aggregation explana-

2 Other people have applied mechanistic ideas outside its original domain, e.g., McKay Illari \& Williamson (2012). 
tions have an extra ingredient. This fits the definition of organisation which is proposed by Arnon Levy and William Bechtel:

When we speak of organization, we do not mean simply the having of some internal structure. Organization, as we see it, involves an internal division of causal labor whereby different components perform different causal roles. A homogenous fluid in a flask has complex internal structure. But (for the most part) its behavior does not involve differential causal roles of components. On this conception, some systems are organized, whereas others are not. More precisely, some systems are more organized than others. (2013, 243)

By rejecting the equation of organisation and internal structure, this characterisation creates the possibility of non-mechanistic micro-explanations: while mechanistic explanations use entities, activities and organisation (and nothing else), there can be explanations which use an extra ingredient: "non-organisational" information about the internal structure of the system. Aggregation explanations as we conceive them fit into this category. ${ }^{3}$

Levy \& Bechtel use "internal division of causal labour" as criterion to distinguish organisation from other information about internal structure. We have proposed a very similar idea in Weber, Van Bouwel and De Vreese (2013, 61-62).

\section{3}

Our aim in this paper is to find out what the surplus value is (if any) of providing unificatory information in micro-explanations of physical laws. Unificatory information is information that provides ontological unification in the sense of Uskali Mäki:

Ontological unification is a matter of redescribing apparently independent and diverse phenomena as manifestations (outcomes, phases, forms, aspects) of one and the same small number of entities, powers, and processes. $(2001,498)$

This characterisation of unificatory information is rather loose, and we will be more specific in subsequent sections. However, ontological unification rules out what Mäki calls derivational unification (2001, 493-4), which aims at an optimal trade-off between either a low number of premises and a high number of conclusions (Michael Friedman) or a low number of derivational patterns and a high number of conclusions (Philip Kitcher).

In order to make the aim of this paper a legitimate one, we have to make two (strongly interconnected) assumptions:

(1) In explanatory texts that provide a micro-explanation of a law, it is possible to separate the information that is required for explaining the target law from additional unificatory information.

(2) Scientists, when writing explanatory texts, can opt to be minimalistic (i.e. only provide information relevant for explaining the target law) or can choose to add unificatory information.

The examples we give in this paper support these assumptions, but at this moment we cannot give any arguments yet.

\footnotetext{
${ }^{3}$ McKay Illari \& Williamson (2012) equate organisation with internal structure. Since we side with Levy $\&$ Bechtel, we do not agree with this.
} 
The method we use exploits these assumptions. We look at examples of explanations of laws. For each case, we construct two possible explanations: a minimal one (without unificatory information) and an elaborate one (with unificatory information). Then we compare the relative merits of these two constructions.

\section{4}

The structure of this paper is as follows. In Section 2 we present an example about the behaviour of mercury thermometers. In Section 3 we analyse this example along the lines explained in 1.3 and go deeper into what "unificatory information" means in this context. In Section 4 we present a second example: the ideal gas law. In Section 5 we analyse this second example and compare the results of this analysis with that of Section 3. Finally, in Section 6 we situate what we have done in the philosophical literature on unification.

\section{Mercury thermometers}

\section{1}

The example we use here is inspired by an example of Carl Hempel $(1965,246)$. A mercury thermometer is rapidly immersed in hot water. We observe a temporary drop of the mercury column, followed by a swift rise. This event can be explained (according to Hempel's DN model) by subsuming it under the following law:

(L) In all mercury thermometers that are rapidly immersed in hot water, the mercury level first drops and then rises.

Hempel uses this law as part of the explanans in his example, because he wants to give an example of an explanation of a particular fact. In our example, the law is the explanandum. Three general laws are relevant for explaining $(\mathrm{L})$ : the law of thermal expansion of fluids, the law of thermal expansion of solids and the law of heat conduction. The law of thermal expansion of fluids is:

(1) $v=v_{\mathrm{o}}+v_{\mathrm{o}} \beta t$

$\left(v\right.$ : final volume; $v_{0}$ : initial volume, $\beta$ : coefficient of expansion of the fluid considered; $t$. rise $(+)$ or fall $(-)$ of the temperature). The law of thermal expansion of solids is:

(2) $l=l_{0}+l_{0} \alpha t$

(l: final length; $1_{0}$ : initial length; $\alpha$ : coefficient of linear expansion of the solid considered). Finally, the law of heat conduction says that the quantity of heat $d \mathrm{Q}$ (joules) which, in a time period $d$ t, flows between any two plane surfaces normal to the direction of heat flow, is given by the equation

(3) $d Q=-\mathrm{K} \cdot \mathrm{A}(\delta \theta / \delta n) d t$

(K: thermal conductivity coefficient (joule $/{ }^{\circ} \mathrm{C} . \mathrm{sec} . \mathrm{m}$ ); $A$ : area of the surfaces; $\delta \theta / \delta n$ : temperature gradient). 
Mercury thermometers consist of glass tubes which are partly filled with mercury. The law of thermal expansion of fluids, when implemented for mercury, gives us the equation:

(4) $v=v_{0}+v_{0} \times 0.00018 \times t_{m}$

( $v$ : final volume of the mercury; $v_{\mathrm{o}}$ : initial volume of the mercury; 0.00018: coefficient of expansion of mercury; $t_{\mathrm{m}}$ : rise of the temperature of the mercury). On the other hand, the tube of the thermometer will expand in accordance with the following equation, which is an implementation of the law of thermal expansion of solids:

(5) $r=r_{o}+r_{0} \times 0.000008 \times t_{g}$

( $r$ : final radius of the tube; $r_{\mathrm{o}}$ : initial radius of the tube; 0.000008: coefficient of linear expansion of glass; $t_{\mathrm{g}}$ : rise of the temperature of the tube). Glass is a bad thermal conductor: its thermal conductivity is small. As a consequence, in a first phase, only the temperature of the tube will rise: $t_{\mathrm{m}}=0$ and thus $v=v_{\mathrm{o}}$. The relation between the level of the mercury and its volume is given by the geometrical formula

(6) $b=v / \pi r^{2}$

( $b$ : height of the mercury column; $v$ : volume of the mercury; $r$ : radius of the tube). From this formula and $\mathrm{v}=\mathrm{v}_{\mathrm{o}}$ we derive that

(7) $h=v_{\mathrm{o}} / \pi r^{2}$

The initial mercury level is given by

(8) $h_{\mathrm{o}}=v_{\mathrm{o}} / \pi r_{\mathrm{o}}^{2}$.

Because the temperature of the tube rises $\left(t_{\mathrm{g}}>0\right), \mathrm{r}$ is larger than $r_{\mathrm{o}}$. The two last equations and $r>r_{o}$ imply that

(9) $b<h_{0}$

In other words: the mercury level drops in a first phase. But as a result of heat conduction, the temperature of the mercury will start to rise after a few instants; the mercury expands (law of thermal expansion of fluids). Because the coefficient of expansion of mercury is large, the expansion will be greater than the extension of the tube. Consequently, the mercury level will rise.

\section{Analysis of the mercury example}

3.1

The explanation in Section 2 fits our definition of a mechanistic explanation: it identifies the components (glass tube, mercury) and describes how they behave (equations (4) and (5)); and equations (6)-(9) are consequences of the organisation of the parts (the most important organisation-fact is that that mercury is inside the glass tube).

\section{2}

Let us now separate the information that is required for explaining the target law from the additional unificatory information. Equations (1) and (2) are not really necessary 
for describing how mercury thermometers work: all we need is (4) and (5), and these can be known independently (by means of experiments with mercury and glass). Similarly, equation (3) is not used in the derivation. Instead, we use its instantiation where $\mathrm{K}$ is substituted for the thermal conductivity coefficient of glass.

In order get a better grip on what is going on here, it is useful to distinguish functional laws from parametric laws. The way we distinguish these types of laws and the examples are based on Ajdukiewicz (1974, 318-23). Let us first look at an example of a functional law:

For all free falling material objects $x$ near the surface of the earth and for all numbers $s$ en $t$ if $s$ is the distance covered by $x$ and $t$ the time during which $x$ is falling, then $s=9.81 \times t^{2} / 2$.

This is Galileo's law. Formally, we can write this as:

$$
\left.(\forall x)(\forall s)(\forall t)\left[(\mathrm{S}(x)=s \wedge \mathrm{T}(x)=t) \rightarrow s=9.81 \times t^{2} / 2\right)\right] .
$$

The general form of functional laws is:

For all objects $x$ in domain $\mathrm{D}$ and all numbers $r_{1}, r_{2}, \ldots, r_{\mathrm{n}}$ : if $\mathrm{M}_{1}(x)=r_{1}, \mathrm{M}_{2}(x)=r_{2}$, $\ldots, \mathrm{M}_{\mathrm{n}}(x)=r_{\mathrm{n}}$, then $\mathrm{F}\left(r_{1}, \ldots, r_{\mathrm{n}}\right)=0$.

An example of a parametric law is:

For all gas samples $x$ with fixed temperature there is a parameter $k$ such that for all numbers $p$ en $v$ : if $p$ is the pressure of $x$ and $v$ its volume at the same moment of time then, $p \times v=k$.

Formally, we can write this as:

$$
(\forall x)(\exists k)[(\forall p)(\forall v)[(\mathrm{P}(x)=p \wedge \mathrm{V}(x)=v) \rightarrow \mathrm{p} \times \mathrm{v}=k] .
$$

The general form of parametric laws is:

For all objects $x$ in domain $\mathrm{D}$ there is a parameter $k$ such that for all numbers $r_{1}, r_{2}, \ldots, r_{\mathrm{n}}:$ if $\mathrm{M}_{1}(x)=r_{1}, \mathrm{M}_{2}(x)=r_{2}, \ldots, \mathrm{M}_{\mathrm{n}}(x)=r_{\mathrm{n}}$, then $\mathrm{F}\left(k, r_{1}, \ldots, r_{\mathrm{n}}\right)=0$.

The explanation presented in Section 2 consists of (i) a derivation of the law to be explained from functional laws about the behaviour of the parts and knowledge about their interaction, and (ii) information (contained in parametric laws) about similar behaviour of related objects. The parametric and functional laws are logically independent. The parametric laws are not derivable from the functional ones because the parametric laws are more general (they are about a larger class of objects); the functional laws are not derivable from the parametric laws because the functional laws are more precise (they contain a specific value for the parameter). As a consequence, there is unificatory information which can be easily separated from the rest of the explanation. The explanation as a whole consists of a part that tells us how things work in the case at hand, and a part that connects this mechanism to potential mechanisms in similar cases. If we delete the latter part, we have a minimal explanation. If we add it, we have an elaborate explanation. In 3.3 we compare the value of these two possible constructions. 
3.3

In our evaluation we use the core idea on explanatory power which Jim Woodward introduced in his book Making Things Happen: "explanation is a matter of exhibiting systematic patterns of counterfactual dependence" $(2003,191)$. He discusses a classic example $(2003,187)$ :

All ravens are black.

$a$ is a raven.

$a$ is black.

He claims that this is not a satisfactory explanation because it ...

... doesn't tell us about the conditions under which raven $a$ would be some other color than black. $(2003,193)$

This fits into his general idea: the argument above tells us that we could have expected that $a$ is black, given that we knew that it was a raven. But expectability is not enough for Woodward: an explanation must also tell how the explanandum would change if the initial conditions would be different. In other words, adequate explanations ...

... locate their explananda within a space of alternative possibilities and show us how which of these alternatives is realized systematically depends on the conditions cited in the explanans. They do this by enabling us to see how, if these initial conditions had been different or had changed in various ways various of these alternatives would have been realized instead. $(2003,191)$

Before we apply this idea to our case, let us give a simple example of a good and a bad explanation. According to Woodward's criterion of explanatory power, the following argument is useless:

(L) All pendula with a length of 1 metre have a period of 2.006 seconds.

(C) This pendulum has a length of 1 metre.

(E) This pendulum has a period of 2.006 seconds.

However, the following argument does have explanatory power:

(L') For all pendula: $\mathrm{P}=2 \pi \cdot \sqrt{\mathrm{L}} / \mathrm{g}$.

(C) This pendulum has a length of 1 metre.

(E) This pendulum has a period of 2.006 seconds.

The law (L') provides counterfactual information: because of its presence the second argument tells us how the period would be different if the length of the pendulum were different. Law $L$ in the first argument does not give that kind of information. In the terminology of Woodward \& Hitchcock (2003) and Hitchcock \& Woodward (2003), (L') is an explanatory generalisation while $\mathrm{L}$ is not explanatory:

Explanatory generalizations allow us to answer what-if-things-had been-different questions: they show us what the value of the explanandum variable depends upon. (Hitchcock \& Woodward 2003 , 183) 
Let us now apply Woodward's view on explanatory power to the mercury example. The minimal explanation gives some counterfactual information: we can calculate what would happen if the expansion coefficients of glass and mercury would be higher or lower than they actually are. The elaborate explanation gives the same counterfactual information, but offers something extra: insight in what would happen if the thermometer would be made of different materials. What if the tube of mercury thermometers would not be made of glass, but of another transparent material? The equations (1)-(3) tell us that similar laws would apply, and that what happens if the differently composed thermometer is immersed in hot water depends on the thermal conductivity and expansion coefficient of the other material.

Woodward not only requires that explanations must answer what-if-things-hadbeen-different questions. He also thinks that explanations which answer more such questions are better than explanations that answer less such questions. This idea is not present in his 2003 book, but clearly present in Hitchcock and Woodward (2003). In our mercury example, both the minimal and the elaborate explanation satisfy Woodward's minimal condition. But the elaborate explanation answers more what-if-thingshad-been-different questions and is therefore better, according to Woodward (and Hitchcock).

\section{4}

We can take the analysis one step further: why is it interesting to have as many answers to what-if-things-had-been-different questions as possible? One reason is practical. If the real situation (the real behaviour we are explaining) is not our ideal, the answers inform us about the possibilities of creating an alternative, more desirable situation. So the unificatory information may have a practical value. Let us clarify this by going deeper into what the unificatory information consists of in this case.

In his definition of ontological unification (see Section 1.3), Mäki uses the word "redescription". This is not what is going on here. Rather, something is added (the parametric laws) to an already existing description. This addition enables us to see the target phenomenon as a manifestation of powers and processes that are also present in other phenomena. In order to see how this additional unificatory information may have a practical value, it is important to note that the model which explains the law is not a complete description of the mechanism that is responsible for the macrobehaviour of the systems. Specific thermometers have properties (e.g. a length and a mass) that are not mentioned in the explanation. The model puts restrictions (in terms of types of entities and organisation) on what the system should look like. All systems which satisfy the constraints exhibit the behaviour described in the explanandum law. The parametric laws enable us to derive what happens in systems that violate the restrictions on the entities but conserve the organisation (thermometers with different materials). So if we are not satisfied with the behaviour of the system, we can use this information to create one that does behave in the way we want. Without the parametric law, we would have no clue about how these modified systems would behave.

Another answer one may give to the question "why is it interesting to have as many answers to what-if-things-had-been-different questions as possible?" is that this 
is what understanding is all about. In other words, we could say that systematic knowledge of counterfactual patterns of dependence is intrinsically valuable because it provides understanding of the world. Such claim is not uncontroversial. Hempel has claimed that science serves two basic human motives: the capacity to predict and control and sheer intellectual curiosity $(1965,333)$. No problem so far. However, he equates this intellectual curiosity with the desire to show that everything could be expected by means of certain laws and initial conditions. In other words, Hempel identifies understanding with expectability. In order to argue that answers to what-if-thingshad-been-different questions are intrinsically valuable, we need a different implementation of the idea of intellectual curiosity. Hempel assumes that we have an inborn, natural desire to try to find out to what extent things that really happened could have been predicted. The Woodwardian version would be that we assume that people have an inborn and natural desire to try to find out what else could have happened and under which conditions.

\section{The ideal gas law}

The ideal gas law (IGL) is about standard, isolated quantities of gases in closed spaces. It claims a proportional relationship between the pressure $P$, the volume $\mathrm{V}$ and the temperature $T$ :

(IGL) $P V=n R T$ in which $\mathrm{R}$ is the ideal gas constant.

For an equal quantity $n$ of gas in a container, the product of pressure $P$ and volume $V$ is proportional to temperature $T$. This proportionality constant, $R$, is the same for all ideal gases and is therefore called the ideal gas constant. To explain why this proportionality holds, we need to understand the underlying machinery. To derive (IGL) from the Kinetic Theory of Gas (KTG) we will use Kuipers' account of explanations of laws (Kuipers 2001, 82-104). According to Kuipers, explanations of laws have different forms, but contain only steps of five types: application steps, aggregation steps, identification steps, correlation steps, and approximation steps. As a rule, these steps occur in this order, but exceptions are possible. Not all explanations contain steps of all types, and some types may occur more than once.

(KTG) assumes the following basic hypotheses:

(a) The ideal gas consists of molecules, and the intermolecular distance in these gases is much larger than the dimensions of the molecule itself. Therefore we can consider those molecules as point masses.

(b) The motion of these molecules is governed by Newton's laws.

(c) The collisions of the molecules with each other or with the walls of the container are in correspondence with Newton's second and third law, and thus considered perfectly elastic.

The first step is to apply these laws to one molecule with a mass $m$ and a velocity $v$ colliding with the wall of the container. This is the application step of (KTG) to (IGL). We consider a cubical box of volume $V$, with side length $L$ and a wall surface $A$ (= $\mathrm{L}^{2}$ ). In that box a molecule will collide with one of the walls at a velocity $v_{x, i}$. We know 
that collisions between opposite walls in the $x$-direction on average will happen in intervals of $\Delta t_{i}=2 \frac{L}{v_{x, i}}$. In that collision the $x$-component of the momentum will be reversed from $m v_{x, i}$ to $-m v_{x, i}$.

(1) This gives us the following force on the wall: $F=\frac{2 m v_{x, i}}{\Delta t_{i}}$

The second step is an aggregation step. By means of a statistical auxiliary hypothesis the individual law is applied to a larger number of molecules. We assume the following auxiliary hypothesis:

(d) The ideal gas is considered a homogeneous distribution of $N$ molecules, which are in constant random motion. $N$ is in the order of $6.022 \times 10^{23}$, the number of Avogadro $N_{A}$.

This results in the following aggregated law:

(2) $F=\sum_{i=1}^{N} \frac{2 m v_{x, i}}{\Delta t_{i}}$.

By using $\Delta t_{i}=2 \frac{L}{v_{x, i}}$ as an identity hypothesis, we can perform a transformation step on (2), which gives us:

(3) $F=\sum_{\mathrm{i}=1}^{N} \frac{\mathrm{mv}_{\mathrm{x}, \mathrm{i}}^{2}}{\mathrm{~L}}=\frac{2}{\mathrm{~L}} \sum_{\mathrm{i}=1}^{N} \frac{\mathrm{mv}^{2} \mathrm{x}, \mathrm{i}}{2}$

To further proceed, we use the following auxiliary hypothesis:

(e) the kinetic energy for one particle in one dimension is defined as: $\mathrm{T}=\frac{1}{2} \mathrm{mv}^{2}$. and apply a second aggregation step:

(4) Since there are $N$ molecules in the container, moving in three dimensions, this gives us a total kinetic energy $T_{\text {tot }}$ of :

$$
T_{\text {tot }}=\frac{1}{2} m \sum_{i=1}^{N}\left(v_{x, i}^{2}+v_{y, i}^{2}+v_{z, i}^{2}\right)=T_{x}+T_{y}+T_{z}
$$

We know apply a identification step, since we assumed that the $N$ molecules are moving in a constant random motion.

(f) Therefore we can say that $T_{x}=T_{y}=T_{z}$, and thus we get:

(5) $T_{\text {tot }}=3 T_{x}$

If we implement this in (3) we get:

(4) $F=\frac{2}{L} \frac{T_{t o t}}{3}$

Now, another auxiliary hypothesis is added:

(g) the pressure $\mathrm{P}$ is defined as a force $F$ exerted on a surface $A$, here the wall of the box: $P=\frac{F}{A}$

Combined with (4), we get another identification step:

(6) $P=\frac{2}{L A} \frac{T_{\text {tot }}}{3}=\frac{2}{3} \frac{T_{\text {tot }}}{V}$ 
By means of the statistical auxiliary hypothesis of the virial theorem, the total kinetic energy of a system is connected with his temperature $T$ in the following formula:

(h) $T_{\text {tot }}=\frac{3}{2} N k T$ in which $k$ is the Boltzmann constant.

We can apply this to (6) and have a third identification step, which gives us:

(7) $P V=N k T$

In another auxiliary hypothesis the Boltzmann constant $k$ is related to the ideal gas constant $R$ as:

(i) $k=\frac{R}{N_{A}}$

As a final result we perform a last identification step on (7) and derive the Ideal Gas Law:

(8) $\quad P V=\frac{N}{N_{A}} R T=n R T$.

\section{Analysis of the IGL explanation}

\section{1}

The explanation fits our definition of a micro-explanation: it identifies the components (walls of the box, molecules) and describes the activities (linear motion of the molecules, collisions). Each of the 6 walls has a specific role in confining the space in which the molecules can move ( 2 boundaries for movement in the $\mathrm{X}$ direction, 2 for the $\mathrm{Y}$ direction and 2 for the $\mathrm{Z}$ direction). This is the organisation of the system. The explanation also uses other information about the internal structure: the randomly distributed direction of motion of the molecules. Therefore, it is not a mechanistic explanation but an aggregation explanation.

\section{2}

Like in the mercury example, we can construct here a minimal explanation and an elaborate one. The minimal one we consider here is the explanation we obtain by taking the result of the application step (equation (1)) as a premise (i.e. by removing the application step). Let us call the result of that move the "minimal IGL explanation" while we call the one presented in Section 4 the "elaborate IGL explanation".

There is an important difference between the IGL case and the thermometer case. In the thermometer case, the additional information in the elaborate explanation did not deductively imply the premises of the minimal explanation. Although the additional information results in more explanatory power, it could be removed without harming the credibility of the explanation: our reasons for accepting the explanation remain intact if we remove it. In the case of the IGL the information that is left out in the minimal explanation provides an argument for believing equation (1): this equation can be logically derived from it. 
This difference creates room for a second advantage that unificatory information may have: providing support for premises we may otherwise doubt. Indeed, if we remove the application step in the explanation in Section 3, it is unclear why we should believe that equation (1) is correct. We certainly cannot do an experiment to support it (that would require a setup where we put exactly one gas molecule in a container and measure the force it exerts on the wall of the container). Our reasons for accepting this premise are theoretical rather than experimental. The elaborate IGL explanation incorporates (at least partially) our theoretical reasons for believing equation (1). The minimal explanation leaves out these reasons. As a consequence, the elaborate IGL explanation is more cogent.

\section{3}

We have now argued that the elaborate explanation is more cogent, but we have not shown yet that it is unificatory. Like in the thermometer example, there is-strictly speaking - no redescription but rather information that is added to an already existing description. Without this additional information, we have no reason to suspect that what we see in gases is a manifestation of the same Newtonian principles we see at work in movements of macroscopic objects. The extra information in the elaborate explanation allows us to see this and at the same time provides a theoretical reason for accepting the explanation.

\section{The role of unification in explanations: a wider picture}

6.1

In Weber, Van Bouwel \& Lefevere (2012) we analyse the role of unification in explanations of particular facts. With respect to this role, we distinguish and discuss five positions:

(1) There is no place for unification in the explanatory process of particular facts. This view is defended in Halonen \& Hintikka (1999). They do not deny the value of unification, but they locate this value outside the explanatory process; unification is a criterion for theory choice.

(2) There are cases in which unification is an explanatory virtue, while the aim of explanation is not unification. In these cases the aim of explanation is to provide information about the causes of the explanandum, but it is better or even required to do this in a unified way.

(3) Unification is an explanatory virtue - in the sense specified in (2) — in all cases of singular explanation.

(4) There are cases in which unification is the main aim of singular explanation (as opposed to merely an explanatory virtue).

(5) Unification is always the main aim of singular explanation (this is the position of Kitcher 1981 and 1989). 
In the paper mentioned above we argue that (2) is correct. A corollary of this is that (1) is refuted. Starting from (2), there are two ways to ascribe a stronger role to unification. We argue that the first way, generalizing from some to all, does not work. In other words, we argue against position (3). The other way, shifting from unification as an explanatory virtue to unification as the main aim of explanation, is also discussed in the paper. We argue that position (4) is correct, while (5) is wrong.

We have mentioned this paper for two reasons. First, we want to draw attention to similar but different work we have done on explanation of particular facts rather than explanation of laws. Second, the overview of possible positions above introduces two contrasts that are important for situating what we have done in the philosophical literature on unification and in the mechanistic tradition in the philosophy of explanation. The contrasts are: (a) all vs. some; and (b) unification as an explanatory virtue vs. unification as the aim of explanation. We use these contrasts in 6.2 to situate our position.

6.2

With respect to explanations of laws, a hard core unificationist will defend the following position:

(SU) When explaining a law, our aim must always be to subsume the law under more general laws and-in this way-to unify our knowledge.

A hard core mechanist holds the following, incompatible position:

(SM) When explaining a law, our aim must always be to provide a model of the mechanism which is responsible for the behaviour at the macro-level.

We think that both positions are wrong. Adopting (SU) implies that one denies the value of mechanistic explanation. Adopting (SM) requires that we deny that the IGL explanation as presented in Section 4 is adequate or adopt a very liberal notion of organisation (cf. McKay Illari \& Williamson 2012). Furthermore, (SM) denies the value of certain inferential explanations of laws (see Gervais \& Weber 2013b for this). We adopt the following views:

(WU) When explaining a law, our aim must sometimes be to subsume the law under more general laws and - in this way-to unify our knowledge.

(WM) When explaining a law, our aim must sometimes be to provide a model of the mechanism which is responsible for the behaviour at the macro-level.

However, the value of what we have done does not depend strongly on these views. What we have done is also important for an adherent of (SM). The reason for this is that we talk about unification as an explanatory virtue, not about unification as an aim of explanation. This will be further discussed in Section 6.3.

Remarkably, what we have done in this paper makes no sense at all for a hard core unificationist who adheres to (SU). From that perspective, we have already made a mistake by positing that scientists try to develop micro-explanations of laws. So from that point of view, our paper rests on a false assumption. 


\section{3}

The paper of Levy \& Bechtel we used in Section 1.2 is about abstraction as an explanatory virtue. The authors argue that-in some contexts_abstract, skeletal models are better explanations than rich models that include a lot of details about the mechanism. A similar point is made in Gervais \& Weber (2013a). The underlying idea of these papers is that mechanistic philosophers (i.e. philosophers who subscribe to (WM) or (SM)) have to think about what makes some mechanistic explanations better than other ones. In other words: they have to think about the explanatory virtues and vices of mechanistic explanations. One axis along which one can compare mechanistic explanations (and other micro-explanations) is richness/abstraction. This axis is explored in the two papers mentioned above. In this paper we have explored a second axis: more or less unification.

\section{Conclusion}

We have argued that, in the context of micro-explanations of laws, providing unificatory information may have two advantages: it can result in higher explanatory power and it can result in more cogent explanations. We do not claim that these advantages are exhaustive: there may be other virtues attached to unification in this context. However, we think we have discovered and described two important ones (i.e., advantages that occur frequently in scientific practice).

In this paper we have approached unification from an epistemologi$\mathrm{cal} /$ methodological angle. Due to space constraints, we did not investigate the possible metaphysical implications of our views. ${ }^{4}$ These issues will be addressed in future work.

\section{REFERENCES}

Ajdukiewicz, Kazimierz. 1974. Pragmatic Logic. Dordrecht: D. Reidel Publishing Company.

Bechtel, William and Adele Abrahamsen. 2005. Explanation: a mechanist alternative, Studies in History and Philosophy of the Biological and Biomedical Sciences 36: 421-441.

Craver, Carl. 2007. Explaining the Brain. Oxford: Clarendon Press.

Feynman Richard, Robert Leighton and Matthew Sands. 2010. The Feynman Lectures on Physics (The New Millennium Edition). Reading: Addison-Wesley Publishing Company.

Gervais, Raoul and Erik Weber. 2013a. Plausibility versus Richness in Mechanistic Models, Philosophical Psychology 26: 139-152.

- 2013b. Inferential Explanations in Biology, Studies in History and Philosophy of Biological and Biomedical Sciences 44: 356-364.

Halonen Ilpo and Jaakko Hintikka. 1999. Unification - It's Magnificent but is it Explanation?, Synthese 120: $27-47$.

Hempel, Carl. 1965. Aspects of Scientific Explanation and other Essays in the Philosophy of Science. New York: Free Press.

Hitchcock Christopher and James Woodward. 2003. Explanatory Generalizations, Part II: Plumbing Explanatory Depth, Noûs 37: 181-199.

${ }^{4}$ For instance-as one of the referees pointed out-we could ask whether our view entails that the best explanations are always those that appeal to fundamental physical properties and laws. 
Kitcher, Philip. 1981. Explanatory Unification, Philosophy of Science 48: 507-531.

- 1989. Explanatory Unification and the Causal Structure of the World. In Scientific Explanation, eds. Philip Kitcher and Wesley Salmon, 410-505. Minneapolis: University of Minnesota Press.

Kuipers, Theo. 2001. Structures in Science. Dordrecht: Kluwer Academic Publishers.

Levy, Arnon and William Bechtel. 2013. Abstraction and the Organization of Mechanisms, Pbilosopby of Science 80: 241-261.

Machamer, Peter, Lindley Darden and Carl Craver. 2000. Thinking About Mechanisms, Philosophy of Science 67: $1-25$.

Mäki, Uskali. 2001. Explanatory Unification. Double and doubtful, Philosophy of the Social Sciences 31: 488506.

Mitchell, Sandra D. 1997. Pragmatic Laws, Philosophy of Science 64: S468-S479.

-. 2000. Dimensions of Scientific Law, Philosophy of Science 67: 242-265.

- 2003. Biological Complexity and Integrative Pluralism. Cambridge: Cambridge University Press.

McKay, Illari Phyllis and Jon Williamson. 2012. What is a mechanism? Thinking about Mechanisms across the Sciences, European Journal for Pbilosophy of Science 2: 119-135.

Weber, Erik, Jeroen Van Bouwel and Leen De Vreese. 2013. Scientific Explanation. Dordrecht: Springer.

Weber, Erik, Jeroen Van Bouwel and Merel Lefevere. 2012. The Role of Unification in Explanations of Facts. In EPS A Philosophy of Science: Amsterdam 2009, eds. H. De Regt, S. Okasha and S. Hartmann, 403-413. Dordrecht: Springer.

Woodward, James. 2003. Making Things Happen. A Theory of Causal Explanation. New York: Oxford University Press.

—. and Christopher Hitchcock. 2003. Explanatory Generalizations, Part I: A Counterfactual Account, Noûs 37: 1-24.

ERIK Weber is full professor of philosophy at Ghent University and director of the Ghent Centre for Logic and Philosophy of Science. His research areas cover philosophy of science and epistemology. He is author of numerous publications in international journals. He recently published a book on Scientific Explanation (Springer, 2013).

Address: Centre for Logic and Philosophy of Science, Ghent University (UGent), Blandijnberg 2, B-9000 Gent, Belgium. Email: erik.weber@ugent.be

Merel Lefevere is teaching assistant and PhD-student at Ghent University and member of the Ghent Centre for Logic and Philosophy of Science. Her research covers philosophy of science and epistemology. She is currently working on a PhD on unification and explanation.

Address: Centre for Logic and Philosophy of Science, Ghent University (UGent), Blandijnberg 2, B-9000 Gent, Belgium. Email: merel.lefevere@ugent.be 